\title{
Minimum recession-compatible subsets of closed convex sets
}

\author{
Yiran He · Jie Sun
}

Received: 18 January 2011 / Accepted: 21 January 2011 / Published online: 9 February 2011

(C) Springer Science+Business Media, LLC. 2011

\begin{abstract}
A subset $B$ of a closed convex set $A$ is recession-compatible with respect to $A$ if $A$ can be expressed as the Minkowski sum of $B$ and the recession cone of $A$. We show that if $A$ contains no line, then there exists a recession-compatible subset of $A$ that is minimal with respect to set inclusion. The proof only uses basic facts of convex analysis and does not depend on Zorn's Lemma. An application of this result to the error bound theory in optimization is presented.
\end{abstract}

Keywords Recession cone $\cdot$ Recession-compatible subset $\cdot$ Error bound

\section{Introduction}

Let $A$ be a nonempty closed convex set in $\mathbb{R}^{n}$. We assume that $A$ is full-dimensional. Otherwise we may equivalently discuss the problem in the subspace that is parallel to the affine hull of $A$. Denote by $A_{\infty}$ the recession cone of $A$. That is,

$$
A_{\infty}=\left\{d \in \mathbb{R}^{n}: x+t d \in A \text { for every } x \in A \text { and all } t \geq 0\right\} .
$$

Some interesting results could be established by using recession cones, see $[1,7,17]$. It can be seen from the definition of the recession cone that the closed convex set $A$ is the (Minkowski) sum of $A$ itself and its recession cone $A_{\infty}[19]$. That is

$$
A=A+A_{\infty}=\left\{x+d: x \in A, d \in A_{\infty}\right\} .
$$

This research is partially supported by National Natural Science Foundation of China under Grant-10701059, and the PROVOST'S CHAIR Grant and the STAR Grant from School of Business, National University of Singapore.

Y. He

Department of Mathematics, Sichuan Normal University, Chengdu, China

J. Sun (西)

Department of Decision Sciences, National University of Singapore, Singapore, Singapore

e-mail: jsun@nus.edu.sg 
In general, if for some subset $B$ of $A, A$ is the sum of $B$ and $A_{\infty}$, i.e.,

$$
A=B+A_{\infty},
$$

then the subset $B$ is said to be recession-compatible with respect to $A$. A recession-compatible subset is minimal with respect to set inclusion if it is contained in all other recessioncompatible subsets. To be concise, here and below, we simply use the word "minimal" and omit "with respect to set inclusion". One can then ask the following question:

- Question. Does there exist a minimal recession-compatible subset of A? If yes, can such a subset be characterized?

We define $A_{p}$ to be the Pareto optimum of $A$ with respect to $-A_{\infty}\left(\right.$ not $\left.A_{\infty}\right)$. That is ([14]),

$$
A_{p}:=\left\{x \in A: A \cap\left(x-A_{\infty}\right)=\{x\}\right\} .
$$

The main purpose of this paper is to show by constructive argument that if $A$ contains no line, then the Pareto optimum $A_{p}$ is the minimal recession-compatible subset with respect to $A$. As its application, we establish a result of error bound, which is a field having been extensively studied; see [4-6,9-11,16,21].

The organization of this paper is as follows. In the next section, we present some basic notations and facts in convex analysis, which are related to the subsequent analysis. Section 3 contains the main results of this paper. We show that if a closed convex set $A$ contains no line, then the minimal recession-compatible subset with respect to $A$ exists. We develop an explicit expression for this set. We present examples to show that, if $A$ contains lines, then the minimal recession-compatible subset with respect to $A$ may not exist. Section 4 provides an application of our result to error bound theory of convex multivalued functions.

\section{Some facts and notations}

We denote $\langle\cdot, \cdot\rangle$ the Euclidean inner product in $\mathbb{R}^{n}$. For a nonempty set $A$ of $\mathbb{R}^{n}, \operatorname{int}(A), \operatorname{cl}(A)$, $\operatorname{bd}(A), \operatorname{ri}(A)$ and $\operatorname{rbd}(A)$ denote the interior, closure, boundary, relative interior, and relative boundary [19] of $A$, respectively. If $x, y \in A$, then $[x, y]$ and $(x, y)$ denote, respectively, the closed and open line segment between $x$ and $y$.

A boundary ray of a closed convex set is a half line contained in the boundary of this convex set. We write $\operatorname{br}(A)$ for the set

$$
\left\{x \in A: \exists d \in A_{\infty}, d \neq 0, x+t d \in \operatorname{bd}(A) \forall t>0\right\} .
$$

We present two examples to help understand the concept of the boundary rays.

Example 1 Let $A$ be the epigraph of the proper convex function $f$ defined by $f(x)=x^{-1}$ for $x \in[1, \infty)$. Then the set of boundary rays $\operatorname{br}(A)$ of $A$ is the set $\{1\} \times[1, \infty)$, a proper subset of the boundary bd $(A)$.

Example 2 Let $A$ be the closed convex set $\{(x, y, z): y \geq|x|, 1 \leq z \leq 2\}$ in $\mathbb{R}^{3}$. Then $\operatorname{br}(A)$ is just the boundary of $A$.

For a linear subspace $L$ in $\mathbb{R}^{n}, L^{\perp}$ denotes the orthogonal complement of $L . \Pi_{L}(\cdot)$ denotes the orthogonal projector onto the linear subspace $L$. It is well known that $\left(L^{\perp}\right)^{\perp}=L$ and every $x \in \mathbb{R}^{n}$ can be decomposed uniquely in the form of $x=\Pi_{L}(x)+\Pi_{L^{\perp}}(x)$.

Lemma 1 ([8]) If $A$ is a convex set in $\mathbb{R}^{n}$ with $\operatorname{int}(A)$ being nonempty, then $t \operatorname{cl}(A)+(1-$ $t) \operatorname{int}(A) \subset \operatorname{int}(A)$ for all $t \in(0,1)$. 
Lemma 2 ([20]) Let $K$ be a nonempty open convex subset of $\mathbb{R}^{n}$. Suppose that $x \in K$ and $y \notin \operatorname{cl}(K)$. Then there exists a point $z$ in $[x, y]$ such that $z \in \operatorname{bd}(K)$.

Lemma 3 Let $A$ be a nonempty unbounded closed convex set. Suppose that $x \in \operatorname{bd}(A)$ but $x \notin \operatorname{br}(A)$. Then for any $d \in A_{\infty} \backslash\{0\}$ and any $t>0, x-t d \notin A$. In particular, if A contains no boundary ray, then $\mathrm{bd}(A)-\left(A_{\infty} \backslash\{0\}\right)$ contains no point of $A$.

Proof If the conclusion does not hold, then there exist $d \in A_{\infty} \backslash\{0\}$ and $t_{0}>0$ such that $x-t_{0} d \in A$. Since $d \in A_{\infty}, x+t d \in A$ for every $t \geq 0$. If $x+t_{1} d \in \operatorname{int}(A)$ for some $t_{1}>0$, then $x \in\left(x-t_{0} d, x+t_{1} d\right) \subset \operatorname{int}(A)$, contradicting $x \in \operatorname{bd}(A)$. Hence $x+t d \in \operatorname{bd}(A)$ for $t \geq 0$ which means that $x \in \operatorname{br}(A)$, contradicting $x \notin \operatorname{br}(A)$.

Lemma 4 Let $A \subset \mathbb{R}^{n}$ be a nonempty closed convex set. If the recession cone $A_{\infty}$ of $A$ contains a linear subspace of which the linear subspace $L$ is the orthogonal complement, then $A \cap L$ is nonempty, and

$$
\operatorname{rbd}(A \cap L)=\operatorname{rbd}(A) \cap L .
$$

In particular, the relative boundary of $A \cap L$ is contained in the relative boundary of $A$.

Proof By Corollary 6.5.1 of [19], it suffices to prove that the set $\operatorname{ri}(A) \cap L$ is nonempty. It can be seen that $A=L^{\perp}+(A \cap L)$ (Page 65 in [19]). Therefore $\operatorname{ri}(A)=L^{\perp}+\operatorname{ri}(A \cap L)$ by [19, Corollary 6.6.2]. Since $\operatorname{ri}(A \cap L) \subset L$ as $L$ is a subspace, it follows that $\operatorname{ri}(A) \cap L=$ $\operatorname{ri}(A \cap L) \neq \varnothing$.

\section{Minimal recession-compatible subset}

Throughout this section, we assume that $A$ is unbounded (If $A$ is bounded, then $A_{\infty}=\{0\}$. Thus $A$ itself is the only recession-compatible subset of $A$ ).

To state the main results, we need to introduce some notations. For $x \in \operatorname{bd}(A)$, let

$$
D(x):=\left\{d \in A_{\infty} \backslash\{0\}: x+t d \in \operatorname{bd}(A) \text { for all } t \geq 0\right\} .
$$

It is easy to see that $D(x) \neq \varnothing$ if and only if $x \in \operatorname{br}(A)$. A point $x \operatorname{in} \operatorname{br}(A)$ is said to be a spine point if for every $d \in D(x), x-t d \notin \operatorname{bd}(A)$ for any $t>0$. We denote $A_{s}$ the set of spine points of $A$; that is,

$$
A_{s}=\{x \in \operatorname{br}(A): x-t d \notin \operatorname{bd}(A), \forall d \in D(x), \forall t>0\} .
$$

It can be seen that for the closed convex sets $A$ in Examples 1 and 2, the sets $A_{s}$ are respectively $\{(1,1)\}$ and $\{(0,0, z): 1 \leq z \leq 2\}$.

Recall that $A_{p}$ is the Pareto optimum set of $A$ with respect to $-A_{\infty}$.

Proposition 1 Let A be a nonempty unbounded closed convex set in $\mathbb{R}^{n}$. Then

$$
A_{p}=(\operatorname{bd}(A) \backslash \operatorname{br}(A)) \cup A_{s} .
$$

Proof Note that $A_{p}=\left\{x: A \cap\left(x-A_{\infty}\right)=\{x\}\right\}$. Let $x \in A_{s}$. If $x \notin A_{p}$, then there exists $d^{*} \in A_{\infty} \backslash\{0\}$ such that $x-d^{*} \in A$. Since $x$ is in $\operatorname{bd}(A)$, it follows that $x-d^{*} \in \operatorname{bd}(A)$ and $x+t d^{*} \in \operatorname{bd}(A)$ for all $t \geq 0$. Thus $d^{*} \in D(x)$ and $x-d^{*} \in \operatorname{bd}(A)$, contradicting $x \in A_{s}$. Therefore $A_{s} \subset A_{p}$, which together with Lemma 3 implies that $(\operatorname{bd}(A) \backslash \operatorname{br}(A)) \cup A_{s} \subset A_{p}$.

Conversely, if $x \in A_{p}$ but $x$ is not in $\operatorname{bd}(A) \backslash \operatorname{br}(A)$, then since $A_{p} \subset \operatorname{bd}(A)$, we have $x \in \operatorname{br}(A)$. It remains to prove that $x \in A_{s}$. If not, then there exists $d \in A_{\infty}$ with $d \neq 0$ such 
that $x+t d \in \operatorname{bd}(A)$ for all $t \geq 0$ and $x-t^{*} d \in \operatorname{bd}(A)$ for some $t^{*}>0$, a contradiction to the assumption of $x \in A_{p}$.

Now we are in a position to state our main results.

Theorem 1 Let A be a nonempty unbounded closed convex set containing no line. Then the Pareto optima set $A_{p}$ is the minimal recession-compatible subset of $A$.

Proof First, we prove that if $B$ is a recession-compatible subset of $A$, then $A_{p}$ is contained in $B$. Indeed, if for some $x \in A_{p}, x \notin B$; then since $B$ is recession-compatible, there exist $d \in A_{\infty}$ with $d \neq 0$ and $y \in B$ such that $x=y+d$. Thus $x-d=y \in A$. Clearly $x-d \in x-A_{\infty}$, hence $x-d \in A \cap\left(x-A_{\infty}\right)$, contradicting $x \in A_{p}$.

Second, we prove that the set $A_{p}$ is recession-compatible with respect to $A$. It suffices to prove that

$$
A \backslash A_{p} \subset A_{p}+A_{\infty} .
$$

Suppose on the contrary, (5) does not hold; i.e.,

$$
\text { there exists } x_{0} \in A \backslash A_{p}, \quad \text { but } x_{0} \notin A_{p}+A_{\infty} \text {. }
$$

Since $x_{0} \in A$ and $A$ contains no line, there exist $x_{1} \in \operatorname{bd}(A)$ and $d_{0} \in A_{\infty} \backslash\{0\}$ such that $x_{0}=x_{1}+d_{0}$. We have $x_{1} \in \operatorname{br}(A) \backslash A_{s}$. (If $x_{1} \notin \operatorname{br}(A) \backslash A_{s}$, then Proposition 1 yields that $x_{1} \in A_{p}$, and hence $x_{0} \in A_{p}+A_{\infty}$, which contradicts (6).) Therefore, the definition (3) of $A_{s}$ yields the existence of $d_{1} \in D\left(x_{1}\right)$ and $t>0$ such that $x_{1}-t d_{1} \in \operatorname{bd}(A)$. Set

$$
t_{1}:=\sup \left\{t>0: x_{1}-t d_{1} \in \operatorname{bd}(A)\right\} .
$$

Since $A$ contains no line, we have $t_{1} \in(0, \infty)$. (If $t_{1}=\infty$, then it can be easily seen that $-d_{1} \in A_{\infty}$. Note that $d_{1} \in D\left(x_{1}\right) \subset A_{\infty} \backslash\{0\}$, thus the set $A$ contains a line with $d_{1}$ as its direction, contradicting the assumption that $A$ contains no line.) It follows that

$$
x_{1}+r d_{1} \in \operatorname{bd}(A) \text { if and only if } r \in\left[-t_{1}, \infty\right) .
$$

Let $x_{2}:=x_{1}-t_{1} d_{1}$. By this inductive procedure, we construct an infinite sequence of vectors $x_{m}, m=1,2, \ldots$, such that

$$
\begin{aligned}
& x_{m+1}:=x_{m}-t_{m} d_{m}, \quad x_{m} \in \operatorname{br}(A) \backslash A_{s}, \text { and } \\
& x_{m}+r d_{m} \in \operatorname{bd}(A) \text { if and only if } r \in\left[-t_{m}, \infty\right), \text { for } m=1,2, \ldots,
\end{aligned}
$$

where $t_{m}:=\sup \left\{t>0: x_{m}-t d_{m} \in \operatorname{bd}(A)\right\}$ and $d_{m} \in D\left(x_{m}\right)$. Suppose that we have generated such $x_{2}, \ldots, x_{j}$ from $x_{1}$. Then we have

$$
\begin{aligned}
& x_{i+1}:=x_{i}-t_{i} d_{i}, \quad x_{i} \in \operatorname{br}(A) \backslash A_{s}, \quad \text { and } \\
& x_{i}+r d_{i} \in \operatorname{bd}(A) \quad \text { if and only if } r \in\left[-t_{i}, \infty\right), \quad \text { for } i=1,2, \ldots, j-1 .
\end{aligned}
$$

We claim that $x_{j} \in \operatorname{br}(A) \backslash A_{s}$. Indeed, if $x_{j} \notin \operatorname{br}(A) \backslash A_{s}$, then (9) and Proposition 1 imply that $x_{j} \in A_{p}$. Noting that

$$
x_{0}=x_{1}+d_{0}=x_{2}+t_{1} d_{1}+d_{0}=\cdots=x_{j}+\sum_{i=1}^{j-1} t_{i} d_{i}+d_{0} \in A_{p}+A_{\infty},
$$

we obtain a contradiction to (6). Since $x_{j} \in \operatorname{br}(A) \backslash A_{s}$, by the definition (3) of $A_{s}$, for some $d_{j} \in D\left(x_{j}\right)$ and some $t>0, x_{j}-t d_{j} \in \operatorname{bd}(A)$. Set

$$
t_{j}:=\sup \left\{t>0: x_{j}-t d_{j} \in \operatorname{bd}(A)\right\} .
$$


Since $A$ contains no line, we have $t_{j} \in(0, \infty)$. It follows that

$$
x_{j}+r d_{j} \in \operatorname{bd}(A) \text { if and only if } r \in\left[-t_{j}, \infty\right) .
$$

Let $x_{j+1}:=x_{j}-t_{j} d_{j}$. Thus the induction step is complete.

On the other hand, it holds that

for each $m \leq n, \quad$ the nonzero vectors $d_{1}, d_{2}, \ldots, d_{m}$ are linearly independent.

We prove (10) by induction. Since $d_{1} \neq 0, d_{1}$ itself is linearly independent. Assume that $d_{1}, d_{2}, \ldots, d_{m-1}$ are linearly independent, we aim to prove that $d_{1}, d_{2}, \ldots, d_{m}$ are linearly independent. If, on the contrary, $d_{1}, d_{2}, \ldots, d_{m}$ are linearly dependent, then there exist real scalars $\lambda_{1}, \lambda_{2}, \ldots, \lambda_{m-1}$ such that

$$
d_{m}=\lambda_{1} d_{1}+\lambda_{2} d_{2}+\cdots+\lambda_{m-1} d_{m-1}
$$

Without loss of generality, assume that $\lambda_{1} \neq 0$.

Case I If $\lambda_{1}>0$, then take positive scalars $r_{2}, r_{3}, \ldots, r_{m-1}$ and take negative scalar $r_{m} \in$ $\left(-t_{m}, 0\right)$ such that

$$
\left\{\begin{array}{l}
t_{m-1}-r_{m} \lambda_{m-1}>0 \\
t_{m-2}-r_{m} \lambda_{m-2}>0 \\
\vdots \\
t_{2}-r_{m} \lambda_{2}>0
\end{array}\right.
$$

The scalar $r_{m}$ satisfying the inequalities (11) does exist because for $i=2, \ldots, m-1$, when $r$ is less than and converges to zero, $t_{i}-r \lambda_{i}$ tends to the positive scalar $t_{i}$. Since $d_{i} \in D\left(x_{i}\right) \subset A_{\infty} \backslash\{0\}$ and $r_{i}>0$ for $i=2, \ldots, m$, we have

$$
x_{i}+r_{i} d_{i} \in A \quad \text { for } i=2, \ldots, m .
$$

Now we want to find nonnegative scalars $\mu_{2}, \mu_{3}, \ldots, \mu_{m}$ with $\mu_{2}+\mu_{3}+\cdots+\mu_{m}=1$, and find $t$ with $t>t_{1}$ such that

$$
\begin{aligned}
x_{1}-t d_{1}= & \mu_{2}\left(x_{2}+r_{2} d_{2}\right)+\mu_{3}\left(x_{3}+r_{3} d_{3}\right)+\cdots \\
& +\mu_{m-1}\left(x_{m-1}+r_{m-1} d_{m-1}\right)+\mu_{m}\left(x_{m}+r_{m} d_{m}\right) .
\end{aligned}
$$

If the solution of (13) exists, then the relation (12), together with the fact that the set $A$ is convex, implies that $x_{1}-t d_{1} \in A$. From Lemma 1, $x_{1}-t d_{1}$ must belong to the boundary of $A$ as $x_{1} \in \operatorname{bd}(A), d_{1} \in D\left(x_{1}\right) \subset A_{\infty} \backslash\{0\}$ and $t>t_{1}>0$. This contradicts (7). Thus we obtain that $d_{1}, d_{2}, \ldots, d_{m-1}, d_{m}$ are linearly independent.

Now it remains to prove that (13) is solvable. Let $c_{m-1}=\left(t_{m-1}-r_{m} \lambda_{m-1}\right) / r_{m-1}$ and

$$
c_{i}=\left[\left(c_{i+1}+\cdots+c_{m-1}\right) t_{i}+\left(t_{i}-r_{m} \lambda_{i}\right)\right] / r_{i}, \quad \text { for } i=2, \ldots, m-2 .
$$

Since each $r_{i}$ and each $t_{i}$ is positive, by use of the inequalities (11), it follows that $c_{2}, \ldots, c_{m-1}$ are all positive. Let $\mu_{m}=\left(1+c_{2}+\cdots+c_{m-1}\right)^{-1}$ and $\mu_{i}=c_{i} \mu_{m}$ for $i=2, \ldots, m-1$, and let $t=t_{1}-\mu_{m} r_{m} \lambda_{1}$. Then $\mu_{2}, \mu_{3}, \ldots, \mu_{m}$ are positive scalars satisfying that $\mu_{2}+\mu_{3}+$ $\cdots+\mu_{m}=1$, and $t>t_{1}$. Now we only need to prove that $\mu_{2}, \mu_{3}, \ldots, \mu_{m}$ and $t$ constructed in this way solve (13). 
Note that $x_{i+1}=x_{i}-t_{i} d_{i}$ for $i=1,2, \ldots, m-1$, and $d_{m}=\lambda_{1} d_{1}+\lambda_{2} d_{2}+\cdots+\lambda_{m-1} d_{m-1}$. It follows that (13) can be reformulated into

$$
\begin{aligned}
x_{1}-t d_{1}= & \mu_{2}\left(x_{1}-t_{1} d_{1}+r_{2} d_{2}\right)+\mu_{3}\left(x_{1}-t_{1} d_{1}-t_{2} d_{2}+r_{3} d_{3}\right)+\cdots \\
& +\mu_{m-1}\left(x_{1}-t_{1} d_{1}-\cdots-t_{m-2} d_{m-2}+r_{m-1} d_{m-1}\right) \\
& +\mu_{m}\left(x_{1}-t_{1} d_{1}-\cdots-t_{m-1} d_{m-1}\right) \\
& +\mu_{m} r_{m}\left(\lambda_{1} d_{1}+\cdots+\lambda_{m-1} d_{m-1}\right)
\end{aligned}
$$

Since $\mu_{2}+\cdots+\mu_{m}=1$, the above expression can be simplified (by cancelling the item $\left.x_{1}\right)$ into

$$
\beta_{1} d_{1}+\beta_{2} d_{2}+\cdots+\beta_{m-1} d_{m-1}=0
$$

where

$$
\left\{\begin{array}{l}
\beta_{1}=t-t_{1}+\mu_{m} r_{m} \lambda_{1}, \\
\beta_{2}=\mu_{m}\left(r_{m} \lambda_{2}-t_{2}\right)-\left(\mu_{3}+\cdots+\mu_{m-1}\right) t_{2}+\mu_{2} r_{2}, \\
\quad \vdots \\
\beta_{m-2}=\mu_{m}\left(r_{m} \lambda_{m-2}-t_{m-2}\right)-\mu_{m-1} t_{m-2}+\mu_{m-2} r_{m-2}, \\
\beta_{m-1}=\mu_{m}\left(r_{m} \lambda_{m-1}-t_{m-1}\right)+\mu_{m-1} r_{m-1} .
\end{array}\right.
$$

It can be seen from the constructions of $\mu_{i}$ and $t$ that

$$
\beta_{1}=\beta_{2}=\cdots=\beta_{m-1}=0 .
$$

Therefore $\mu_{2}, \mu_{3}, \ldots, \mu_{m}$ and $t$ constructed in this way do solve (13). Thus the claim (iv) is true for Case I.

Case II If $\lambda_{1}<0$, then take sufficiently small $r_{m}>0$ such that (11) is satisfied, and take positive scalars $r_{2}, \ldots, r_{m-1}$. The remaining proof is similar to Case I and hence we omit it.

Up to this point we have shown that (10) is true. This implies that $\left\{x_{m}\right\}$ generated as in (8) would not be an infinite sequence, since the space is $n$ dimensional and since the construction of $x_{n+1}$ needs a vector $d_{n}$ having the properties as shown in (8). Thus, we obtain a contradiction, which shows that (6) should not be true, and hence the proof is complete. ${ }^{1}$

In what follows, we allow the closed convex set $A$ to contain lines. In this case, $A$ may have no minimal recession-compatible subset as shown by the following example. However, some similar results to Theorem 1 can be obtained.

Example 3 Let $A$ be the closed convex set $\left\{(x, y) \in \mathbb{R}^{2}: y \geq 1\right\}$, and let $B_{1}=\{(1,1)\}$ and $B_{2}=\{(2,1)\}$. Then $A_{\infty}=\{(x, y): x \in \mathbb{R}, y \geq 0\}$. We have $A=B_{1}+A_{\infty}=B_{2}+A_{\infty}$, i.e., $B_{1}$ and $B_{2}$ are recession-compatible subsets of $A$, and $B_{1} \cap B_{2}=\emptyset$.

The singletons $B_{1}$ and $B_{2}$ are recession-compatible with respect to $A$, if $A$ has a minimal recession-compatible subset, then this minimal subset should be contained in $B_{1}$ and $B_{2}$ by definition. Thus, $B_{1} \cap B_{2}$ should be nonempty. However, $B_{1} \cap B_{2}$ is actually an empty set. This contradiction shows that the minimal recession-compatible subset of this set does not exist.

${ }^{1}$ After this paper was finished, we noticed that there are other proofs for Theorem 1 such as by Zorn's lemma and by separation theorem of convex sets. Different from these arguments, our proof is constructive. 
Theorem 2 Let $A \subset \mathbb{R}^{n}$ be a nonempty closed convex subset. If the recession cone $A_{\infty}$ of $A$ is a linear subspace, then $A \cap\left(A_{\infty}\right)^{\perp}$ is a recession-compatible subset of $A$. If $B$ is recession-compatible with respect to $A$, then $A \cap\left(A_{\infty}\right)^{\perp}$ is contained in the projection of $B$ onto $\left(A_{\infty}\right)^{\perp}$.

Proof Set $L=\left(A_{\infty}\right)^{\perp}$. Since $A_{\infty}$ is a linear subspace, it follows from [19, p. 65.] that $A \cap L$ is recession-compatible subset of $A$.

Let $B$ be any recession-compatible subset of $A$. Given $x \in A \cap L$, since $B$ is recessioncompatible with respect to $A$, there exist $y \in B$ and $z \in A_{\infty}$ such that $x=y+z$. Applying orthogonal decomposition, we have $x=\Pi_{L}(y)+\Pi_{L^{\perp}}(y)+z$, and hence

$$
x-\Pi_{L}(y)=\Pi_{L^{\perp}}(y)+z .
$$

However, $x-\Pi_{L}(y) \in L$ and $\Pi_{L^{\perp}}(y)+z \in L^{\perp}$ as $z \in A_{\infty}=L^{\perp}$. It follows from (14) and $L \cap L^{\perp}=\{0\}$ that $x-\Pi_{L}(y)=0$. Thus $x=\Pi_{L}(y) \in \Pi_{L}(B)$. Therefore $A \cap L \subset \Pi_{L}(B)$.

Theorem 3 Let A be a nonempty closed convex set. Suppose $A_{\infty}$ is not a linear subspace and $L$ is the orthogonal complement of the largest linear subspace contained in $A_{\infty}$. Then $A \cap L$ is nonempty and $(A \cap L)_{p}$ is a recession-compatible subset of $A$. Furthermore, if $B$ is any recession-compatible subset of $A$, then $(A \cap L)_{p} \subset \Pi_{L}(B)$.

Proof By Lemma 4, $A \cap L$ is nonempty. Since $L^{\perp}$ is the largest linear subspace contained in $A_{\infty}$ and since $A_{\infty}$ is not a linear subspace, $A \cap L$ contains no line and is unbounded. Applying Theorem 1, we have that the set $(A \cap L)_{p}$ is recession-compatible with respect to $A \cap L$. Note that $A=(A \cap L)+L^{\perp}\left[19\right.$, p. 65]. Since $(A \cap L) \cap L^{\perp}=\{0\}$, it follows from Corollary 9.1.2 of [19] that $A_{\infty}=\left((A \cap L)+L^{\perp}\right)_{\infty}=(A \cap L)_{\infty}+L^{\perp}$. Thus

$$
A=(A \cap L)_{p}+A_{\infty},
$$

which implies that the set $(A \cap L)_{p}$ is a recession-compatible subset of $A$.

Now it remains to prove the second assertion. Since $A \cap L$ is nonempty and contains no line as verified above, it follows from Theorem 1 that if $\Pi_{L}(B)$ is recession-compatible with respect to $A \cap L$, then $(A \cap L)_{p}$ is actually contained in $\Pi_{L}(B)$. Therefore it suffices to prove

$$
A \cap L=\Pi_{L}(B)+(A \cap L)_{\infty},
$$

equivalently, to prove $\left(B+A_{\infty}\right) \cap L=\Pi_{L}(B)+\left(A_{\infty} \cap L\right)$. Let $x \in\left(B+A_{\infty}\right) \cap L$. Then there exist $y \in B$ and $z \in A_{\infty}$ such that $x=y+z$. Since $x \in L$ and since the orthogonal projection operator is linear, it follows that

$$
\begin{aligned}
& x=\Pi_{L}(y)+\Pi_{L}(z), \\
& 0=\Pi_{L^{\perp}}(y)+\Pi_{L^{\perp}}(z) .
\end{aligned}
$$

Since $z \in A_{\infty}$, we have $x+t z \in A$ for any $t>0$, which, together with the fact that $z=\Pi_{L}(z)+\Pi_{L^{\perp}}(z)$, implies that for any $t>0$,

$$
\begin{aligned}
x+t \Pi_{L}(z) \in-t \Pi_{L^{\perp}}(z)+A & =t \Pi_{L^{\perp}}(y)+A \\
& =\Pi_{L^{\perp}}(t y)+A \subset A_{\infty}+A=A,
\end{aligned}
$$

in which the first equality follows from (16), and the inclusion relation holds as $L^{\perp}$ is contained in $A_{\infty}$. This shows that $\Pi_{L}(z) \in A_{\infty}$. By (15), it follows that

$$
x \in \Pi_{L}(B)+\left(A_{\infty} \cap L\right) .
$$

Therefore $\left(B+A_{\infty}\right) \cap L \subset \Pi_{L}(B)+\left(A_{\infty} \cap L\right)$. 
Now we prove the converse inclusion. Let $x \in \Pi_{L}(B)+\left(A_{\infty} \cap L\right)$. Then there exists $y \in B$ such that $x-\Pi_{L}(y) \in A_{\infty}$. It follows that

$$
\begin{gathered}
x-y=x-\Pi_{L}(y)-\Pi_{L^{\perp}}(y) \in A_{\infty}-\Pi_{L^{\perp}}(y) \\
\subset A_{\infty}-L^{\perp}=A_{\infty}+L^{\perp} \subset A_{\infty} .
\end{gathered}
$$

Thereby $x \in y+A_{\infty} \subset B+A_{\infty}$. Clearly $x \in L$, we have

$$
\Pi_{L}(B)+\left(A_{\infty} \cap L\right) \subset\left(B+A_{\infty}\right) \cap L .
$$

This completes the proof.

Example 4 Assume the closed convex set $A$ is defined by a convex quadratic function, namely, $A:=\left\{x \in \mathbb{R}^{n}: \frac{1}{2} x^{T} H x+b^{T} x+c \leq 0\right\}$, where $H$ is a symmetric positive semidefinite $n \times n$ matrix, $b \in \mathbb{R}^{n}$, and $c \in \mathbb{R}$. If $H$ is nonsingular, then the boundary ray of $A$ does not exist, because of $A_{\infty}=\left\{d: H d=0, b^{T} d \leq 0\right\}$. If $H$ is singular and $\left\{d: H d=0, b^{T} d=0\right\}=\{0\}$, then the boundary ray of $A$ does not exist. In these two cases, the minimal recession-compatible subset of $A$ is the boundary of $A$.

If $H$ is singular and $\left\{d: H d=0, b^{T} d=0\right\} \neq\{0\}$, then $A_{\infty}$ contains a linear subspace. Thus Theorem 3 is applicable.

\section{An application to error bound analysis}

Let $X$ be a Banach space and $\Gamma: \mathbb{R}^{n} \rightarrow X$ be a convex multivalued function (multifunction for short) with closed graph. A multifunction $\Gamma$ is said to be convex if

$$
\lambda \Gamma(x)+(1-\lambda) \Gamma(y) \subset \Gamma(\lambda x+(1-\lambda) y), \quad \text { for } x, y \in \mathbb{R}^{n}, \lambda \in(0,1) .
$$

Given $x_{0} \in X$ with $\Gamma^{-1}\left(x_{0}\right)$ nonempty, we consider to find a positive scalar $\gamma$ such that

$$
d\left(x, \Gamma^{-1}\left(x_{0}\right)\right) \leq \gamma d\left(x_{0}, \Gamma(x)\right), \quad \text { for all } x \in \mathbb{R}^{n},
$$

where $d(\cdot, \cdot)$ stands for the distance function. If the above expression holds only for every point in some neighbourhood of the point $x_{0}$, we say that local error bound holds around $x_{0}$. Error bound for convex multifunction has been discussed in the literature; see [13,15,18]. Actually, the concept of recession-compatible subset was firstly used in [15]. After that, global error bound was analyzed in terms of the so-called recession core in [22].

We will assume throughout this section that $\Gamma^{-1}\left(x_{0}\right)$ is unbounded because [13] presented detailed discussion on the case when $\Gamma^{-1}\left(x_{0}\right)$ is bounded.

Theorem 4 Let $\Gamma: \mathbb{R}^{n} \rightarrow X$ be a convex multifunction with closed graph and $\Gamma^{-1}\left(x_{0}\right) \neq \emptyset$. Suppose that $\Gamma^{-1}\left(x_{0}\right)$ is unbounded and contains no line. If $\gamma>0$ is a constant such that local error bound holds around every point in the minimal recession-compatible subset of $\Gamma^{-1}\left(x_{0}\right)$, then global error bound (17) holds with the same constant $\gamma$.

Proof In view of Theorem 1, the minimal recession-compatible subset of $\Gamma^{-1}\left(x_{0}\right)$ is nonempty, denoted by $\mathcal{M}$. Let $x \notin \Gamma^{-1}\left(x_{0}\right)$. We divide the discussion into two cases:

(i) If the projection $\bar{x}$ of $x$ onto $\Gamma^{-1}\left(x_{0}\right)$ belongs to $\mathcal{M}$, then

$$
\begin{aligned}
d\left(x, \Gamma^{-1}\left(x_{0}\right)\right) & \leq\|x-\bar{x}-\lambda(x-\bar{x})\|+d\left(\bar{x}+\lambda(x-\bar{x}), \Gamma^{-1}\left(x_{0}\right)\right) \\
& =(1-\lambda)\|x-\bar{x}\|+d\left(\bar{x}+\lambda(x-\bar{x}), \Gamma^{-1}\left(x_{0}\right)\right) \\
& =(1-\lambda) d\left(x, \Gamma^{-1}\left(x_{0}\right)\right)+d\left(\bar{x}+\lambda(x-\bar{x}), \Gamma^{-1}\left(x_{0}\right)\right),
\end{aligned}
$$


which implies that

$$
d\left(x, \Gamma^{-1}\left(x_{0}\right)\right) \leq \frac{1}{\lambda} d\left(\bar{x}+\lambda(x-\bar{x}), \Gamma^{-1}\left(x_{0}\right)\right) .
$$

On the other hand, since local error bound holds with $\gamma>0$ around $\bar{x}$, there exists $\lambda \in(0,1)$ such that $\bar{x}+\lambda(x-\bar{x})$ belongs to the neighbourhood of $\bar{x}$ on which the local error bound around $\bar{x}$ holds. It follows that

$$
d\left(\bar{x}+\lambda(x-\bar{x}), \Gamma^{-1}\left(x_{0}\right)\right) \leq \gamma d\left(x_{0}, \Gamma(\bar{x}+\lambda(x-\bar{x}))\right) \leq \lambda \gamma d\left(x_{0}, \Gamma(x)\right),
$$

where the second inequality follows from the convexity of $\Gamma$.

The last two expressions yield that

$$
d\left(x, \Gamma^{-1}\left(x_{0}\right)\right) \leq \gamma d\left(x_{0}, \Gamma(x)\right) .
$$

(ii) If the projection $\bar{x} \notin \mathcal{M}$, then there is $a \in \mathcal{M}, c \in\left(\Gamma^{-1}\left(x_{0}\right)\right)_{\infty}$ with $c \neq 0$ such that $\bar{x}=a+c$. We claim that for $\lambda \in(0,1)$,

$$
d\left(a+\lambda(x-a), \Gamma^{-1}\left(x_{0}\right)\right)=\lambda\|x-a-c\| .
$$

Thus

$$
\begin{aligned}
d\left(x, \Gamma^{-1}\left(x_{0}\right)\right) & =\|x-\bar{x}\|=\|x-a-c\| \\
& =\frac{1}{\lambda} d\left(a+\lambda(x-a), \Gamma^{-1}\left(x_{0}\right)\right) \\
& \leq \frac{1}{\lambda} \gamma d\left(x_{0}, \Gamma(a+\lambda(x-a)) \text { for small enough } \lambda \in(0,1)\right. \\
& \leq \gamma d\left(x_{0}, \Gamma(x)\right),
\end{aligned}
$$

where the first inequality follows from the fact that local error bound holds around $a \in \mathcal{M} \subset \Gamma^{-1}\left(x_{0}\right)$ while the second one from the convexity of $\Gamma$.

Now we prove the claim. Since $\bar{x}=a+c$ is the projection of $x$ onto $\Gamma^{-1}\left(x_{0}\right)$, we have

$$
\langle x-(a+c), y-(a+c)\rangle \leq 0, \text { for all } y \in \Gamma^{-1}\left(x_{0}\right) .
$$

Noticing that $c \in \Gamma^{-1}\left(x_{0}\right)_{\infty}$, we have for any $y \in \Gamma^{-1}\left(x_{0}\right)$ and $\lambda \in(0,1), y+(1-\lambda) c$ belongs to $\Gamma^{-1}\left(x_{0}\right)$. It follows that

$$
\langle\lambda(x-(a+c)), y-(a+\lambda c)\rangle \leq 0, \text { for all } y \in \Gamma^{-1}\left(x_{0}\right) \text { and } \lambda \in(0,1) .
$$

This implies that $a+\lambda c$ is the projection of $a+\lambda(x-a)$ onto $\Gamma^{-1}\left(x_{0}\right)$, and hence the claim follows.

Remark 1 Theorem 4 requires that the constant $\gamma$ does not depend on the points in the minimal recession-compatible subset of $\Gamma^{-1}\left(x_{0}\right)$. If on the contrary $\gamma$ is dependent on $x$, then the conclusion does not generally hold. We can see this point by letting $x_{0}=0$ and $\Gamma(x)=g(x)+\mathbb{R}_{+}^{n}$ with $g: \mathbb{R}^{\ell} \rightarrow \mathbb{R}^{n}$ and $g_{i}: \mathbb{R}^{\ell} \rightarrow \mathbb{R}(i=1, \ldots, n)$ being differentiable convex functions. That is, we consider convex differentiable inequalities system

$$
g_{1}(x) \leq 0, \ldots, g_{n}(x) \leq 0 .
$$

Li [12] proved that for the above differentiable convex inequalities system, the existence of local error bounds at every feasible point $x$ with $\gamma$ dependent on $x$ is equivalent to the so-called basic constraint qualification. However, the basic constraint qualification is not enough to ensure that global error bound exists; see Theorem 10 in [16]. 
Remark 2 From the proof of Theorem 4, it can be seen that if $B$ is any recession-compatible subset of $\Gamma^{-1}\left(x_{0}\right)$ and local error bound holds with the same constant $\gamma$ around every point in $B$, then global error bound (17) holds. It is therefore desirable that the recession-compatible subset $B$ is as small as possible with respect to set inclusion, which motivates the application of the minimal recession-compatible subset.

Similar to Theorems 1 and 3 , if $\Gamma^{-1}\left(x_{0}\right)$ contains line, one can state a global error bound result similar to Theorem 4 as well. For brevity we omit the discussion. The interested reader may refer to [22, Section 5], which obtained global error bound by assuming local error bound on a recession core. Note that we do not require the recession-compatible subset to be convex.

An interesting topic of future research would be the case of $A=\{x: g(x) \leq 0\}$, where $g$ is a certain function. In the specific case of $g$ being a convex polynomial, some recession properties have been derived in [2], for recent advances see [3,10,21]. Another interesting question is whether a similar result is valid for the strong conical hull intersection property. Namely, let $C$ and $B$ be two closed convex sets and let $A$ be the minimal recession-compatible set of $C \cap B$. We would like to know whether

$$
N_{C \cap B}(x)=N_{C}(x)+N_{B}(x) \forall x \in A \Rightarrow N_{C \cap B}(x)=N_{C}(x)+N_{B}(x) \forall x \in C \cap B,
$$

where $N_{S}(x)$ denotes the convex normal cone of $S$ at $x$.

Acknowledgments The authors are in debt to Professor K.L. Teo of Curtin University, Professor S. Wang of University of Western Australia, and three referees for their insightful comments.

\section{References}

1. Addi, K., Adly, S., Goeleven, D., Saoud, H.: A sensitivity analysis of a class of semi-coercive variational inequalities using recession tools. J. Global Optim. 40, 7-27 (2008)

2. Auslender, A., Teboulle, M.: Asymptotic Cones and Functions in Optimization and Variational Inequalities. Springer Monographs in Mathematics. Springer, New York (2003)

3. Belousov, E.G., Klatte, D.: A Frank-Wolfe type theorem for convex polynomial programs. Comput. Optim. Appl. 22, 37-48 (2002)

4. Burke, J.V., Deng, S.: Weak sharp minima revisited, Part I: basic theory. Control Cyber. 31, 439-470 (2002)

5. Deng, S.: Computable error bounds for convex inequality systems in reflexive Banach spaces. SIAM J. Optim. 7, 274-279 (1997)

6. Deng, S.: Perturbation analysis of a condition number for convex inequality systems and global error bounds for analytic systems. Math. Program. 83, 263-276 (1998)

7. Deng, S., Hu, H.: Computable error bounds for semidefinite programming. J. Global Optim. 14, 105-115 (1999)

8. Holmes, R.B.: Geometric Functional Analysis and its Applications. Springer, New York (1975)

9. Klatte, D., Li, W.: Asymptotic constraint qualifications and global error bounds for convex inequalities. Math. Program. 84, 137-160 (1999)

10. Li, G.: On the asymptotic well behaved functions and global error bound for convex polynomials. SIAM J. Optim. 20, 1923-1943 (2010)

11. Li, G., Ng, K.F.: Error bounds of generalized D-gap functions for nonsmooth and nonmonotone variational inequality problems. SIAM J. Optim. 20, 667-690 (2009)

12. Li, W.: Abadie's constraint qualification, metric regularity, and error bounds for differentiable convex inequalities. SIAM J. Optim. 7, 966-978 (1997)

13. Li, W., Singer, I.: Global error bounds for convex multifunctions and applications. Math. Oper. Res. 23, 443-462 (1998)

14. Luc, D.T.: Theory of vector optimization, Lecture Notes in Economics and Mathematical Systems, vol.319. Springer, Berlin (1989)

15. Ng, K.F., Zheng, X.Y.: Characterizations of error bounds for convex multifunctions on Banach spaces. Math. Oper. Res. 29, 45-63 (2004) 
16. Pang, J.-S.: Error bounds in mathematical programming. Math. Program. 79, 299-332 (1997)

17. Pardalos, P.M., Rassias, T.M., Khan, A.A. (eds.): Nonlinear Analysis and Variational Problems. Springer, Berlin (2010)

18. Robinson, S.M.: Regularity and stability for convex multivalued functions. Math. Oper. Res. 1, 130 $143(1976)$

19. Rockafellar, R.T.: Convex Analysis. Princeton University Press, Princeton (1970)

20. Stoer, J., Witzgall, C.: Convexity and Optimization in Finite Dimensions. Vol. I, Springer, New York (1970)

21. Yang, W.H.: Error bounds for convex polynomials. SIAM J. Optim. 19, 1633-1647 (2008)

22. Zheng, X.Y., Ng, K.F.: Metric subregularity and constraint qualifications for convex generalized equations in Banach spaces. SIAM J. Optim. 18, 437-460 (2007) 